\title{
Review: Observations
}

\section{Multi-frequency Observations of Highly Variable Relativistic Jets}

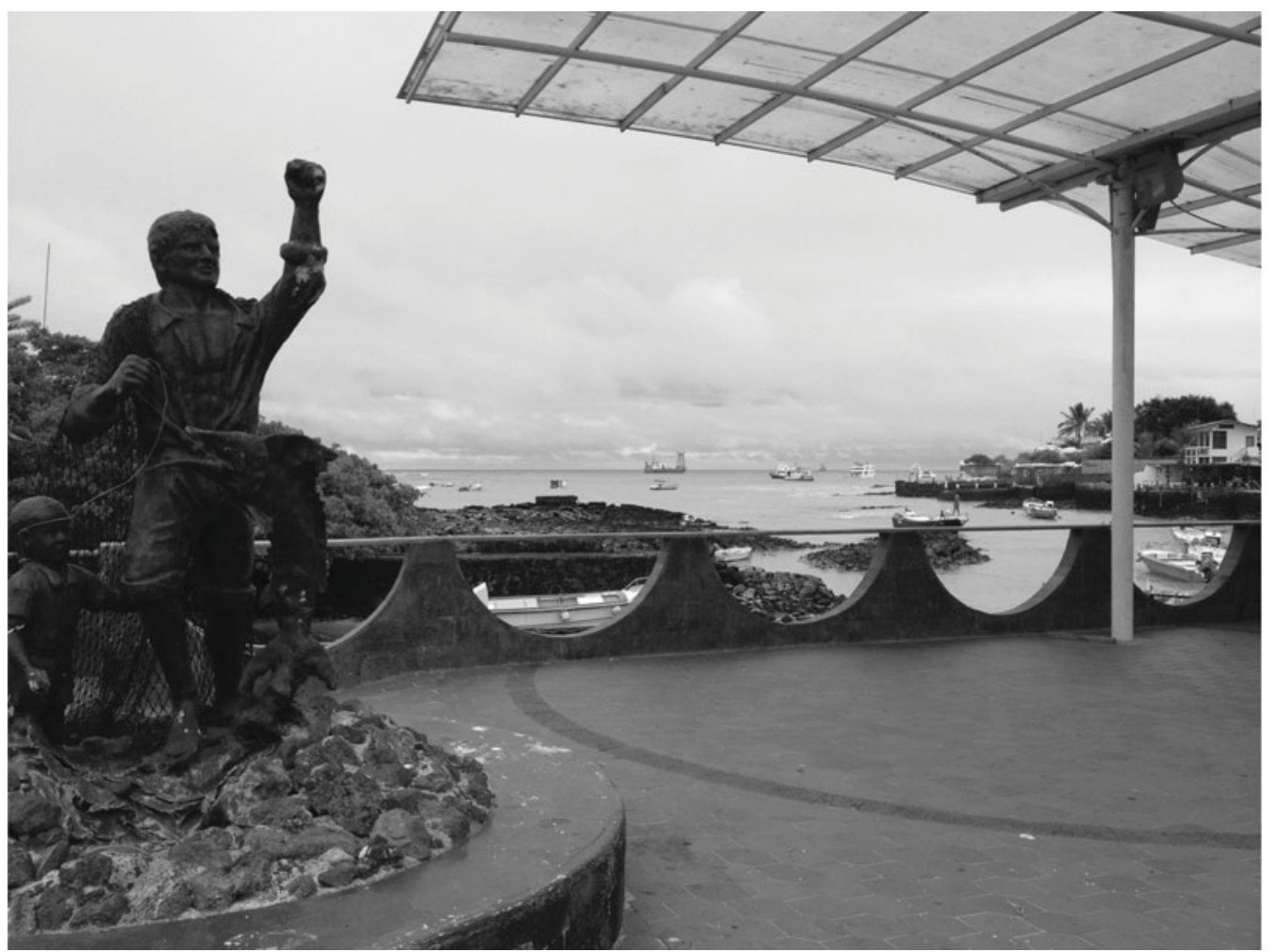

View of Pelican Bay from Av. Charles Darwin 


\title{
Observational aspects of AGN jets at high energy
}

\author{
Jun Kataoka \\ Research Institute for Science and Engineering, Waseda University, \\ 3-4-1 Okubo, Shinjuku, Tokyo, Japan \\ email: kataoka. jun@waseda.jp
}

\begin{abstract}
For the last two decades, significant and dramatic progress has been made in understanding astrophysical jet sources, particularly in the X-ray and gamma-ray energy bands. For example, the Chandra X-ray observatory reveals a number of AGN jets extending from kpc to Mpc scales. More recently, the Fermi Gamma-ray Space Telescopes launched in 2008 started monitoring the gamma-ray sky with excellent sensitivity of about ten times greater than that of EGRET onboard CGRO, and has detected more than 2,000 sources (mostly AGNs) as of 2014. Moreover, Fermi-LAT has discovered gamma-ray emissions not only from blazars but from a dozen radio galaxies not previously known to emit gamma-rays. Closer to home, the Fermi-bubbles were discovered to extend 50 degrees above and below the Galactic center. These large scale diffuse gamma-ray structures are similar in structure to AGN lobes such as those seen in Cen A and provide evidence for past activity in our Galactic center. In this review, I will first summarize recent highlights of large scale jets in radio galaxies, specifically resolved by the Chandra X-ray observatory. Next I will move on to the gamma-ray sky to present some highlights from Fermi-LAT observations of "misaligned" blazars, namely radio galaxies. I will discuss a unification scheme connecting blazars and misaligned radio galaxies. In the last part, I will also briefly comment on recent multiband observations of the Fermi-bubble and possible impacts on the AGN jet physics in the near future.
\end{abstract}

Keywords. galaxies: active, radio continuum: galaxies, X-rays: galaxies, gamma rays: observations

\section{Introduction}

A long-debated problem in understanding of supermassive black holes (SMBHs) is the unification of different types of active galactic nuclei (AGNs). It has been widely argued that the difference between type 1 and type 2 AGNs may be explained by simple geometrical effects involving anisotropic obscuration of the active center viewed at different inclination angles (Antonucci 1993). Also, the accretion rate was claimed to play an important role, that can account for different emission spectra and power outputs of highand low-power AGNs (see an early discussion on this issue by Fabian \& Rees 1995, Lasota 1996, and the more recent one in Ghisellini et al. 2009). The other relevant parameter may also be the mass of the SMBH itself, as proposed to understand difference between narrow-line Seyfert 1 and "regular" Seyfert 1 galaxies (e.g., Pogge 2000, Komossa 2008).

However, none of these can account for the dichotomy between radio-loud AGNs and radio-quiet AGNs. One may therefore seek the fundamental difference between AGNs producing luminous radio-emitting outflows (i.e., jets) and those lacking such outflows in yet another parameter of the accretion disk/black hole system. One possibility is that, radio-loud AGNs harbor rapidly spinning SMBHs with rotational energy extracted electromagnetically via the Blandford \& Znajek (1977) mechanism, and converted to the kinetic luminosity of relativistic jets. This so-called spin paradigm (Blandford 1990) has 

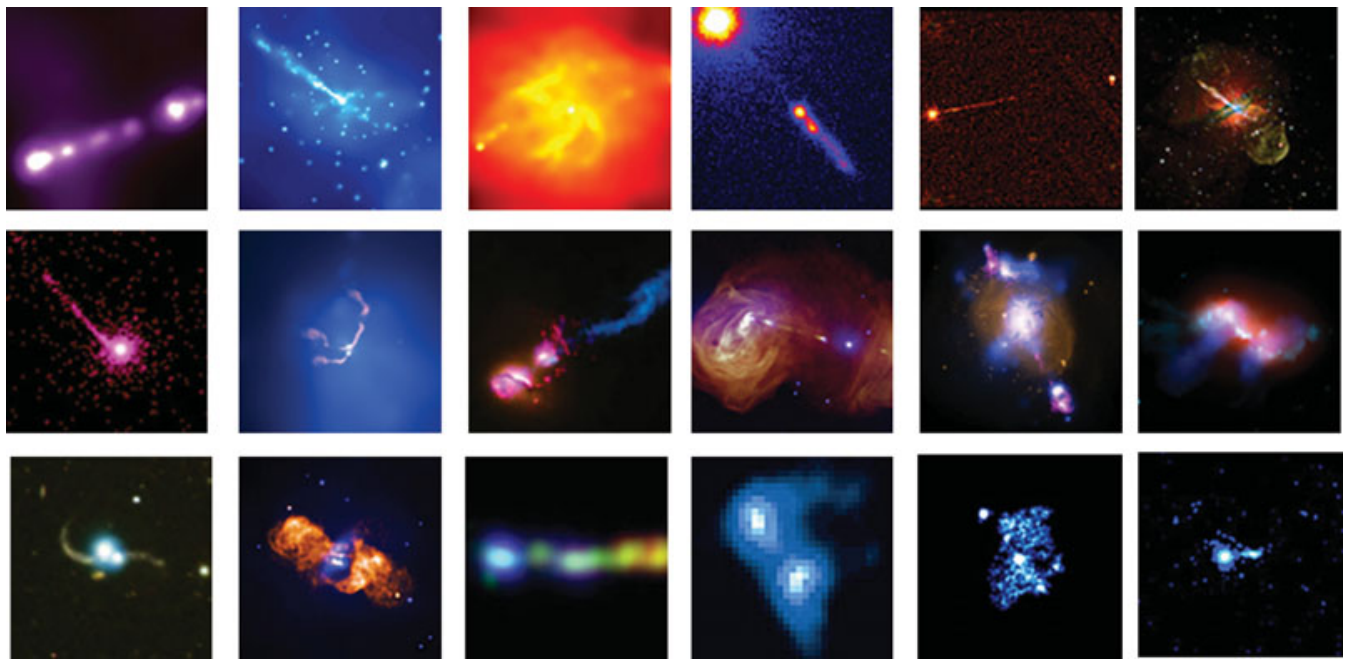

Figure 1. Example images of large scale jets in radio galaxies seen by Chandra X-ray observatory. All the images are collected from NASA's Chandra home page; http://chandra. harvard.edu/photo/category/quasars.html

recently been claimed to be after some modifications, by several observational findings and theoretical investigations (e.g., Koide et al. 2002, Sikora et al. 2007, Tchekhovskoy et al. 2010; see also Garofalo 2009, Maraschi et al. 2012). Interestingly, new deep radio surveys indicate that the radio loudness parameter shows a continuous distribution rather than a sharp division between radio-loud and radio-quiet AGNs. Moreover, unresolved non-thermal radio emission and jet-like structures have been discovered in classes of AGNs considered previously as "radio-quiet," i.e., Seyfert galaxies, although the jets in such systems are non-relativistic and weak when compared to the jets found in "classical' radio galaxies and quasars (e.g., Ulvestad \& Wilson 1989, Kukula et al. 1995, Thean et al. 2001, Middelberg et al. 2004 and references therein). Yet, there is a substantial difference in the distribution of the jet production efficiency between disk-hosted and elliptical hosted AGNs (Sikora et al. 2007).

From a morphological point of view, extragalactic jets constitute the longest collimated structures in the universe. It has been suggested that geometrical effects play again a major role to understand radio-loud AGNs which are observed at small viewing angles to the jet axis (blazars) and those AGNs which are inclined at larger viewing angles (e.g., radio galaxies) (see, e.g., Barthel 1989, Urry \& Padovani 1995). They transport huge amounts of energy from the nuclei of active galaxies out to $\mathrm{kpc}$ or Mpc distances, significantly affecting the properties of the surrounding intracluster/intergalactic medium. Jets have been extensively studied in the radio domain on different scales since the very beginning of the development of modern radio interferometers (Bridle \& Perley 1984, Begelman et al. 1984). More recently, the excellent spatial resolution of the Chandra X-Ray Observatory (and of other X-ray satellites like $X M M$-Newton) has allowed us to image large-scale structures in powerful extragalactic radio sources at X-ray frequencies as well, and thus has opened a new era in studying the high-energy emission of these objects (see, for a review, e.g., Harris \& Krawcynski 2002, 2006; Kataoka \& Stawarz 2005; Croston et al.2005; Massaro et al. 2011, 2012).

Even at higher energy, Fermi Gamma-ray Space Telescope and various ground-based Cherenkov telescopes like HESS, MAGIC, and VERITAS provide new opportunities to study the gamma-ray emission from different types of extragalactic sources - not only 

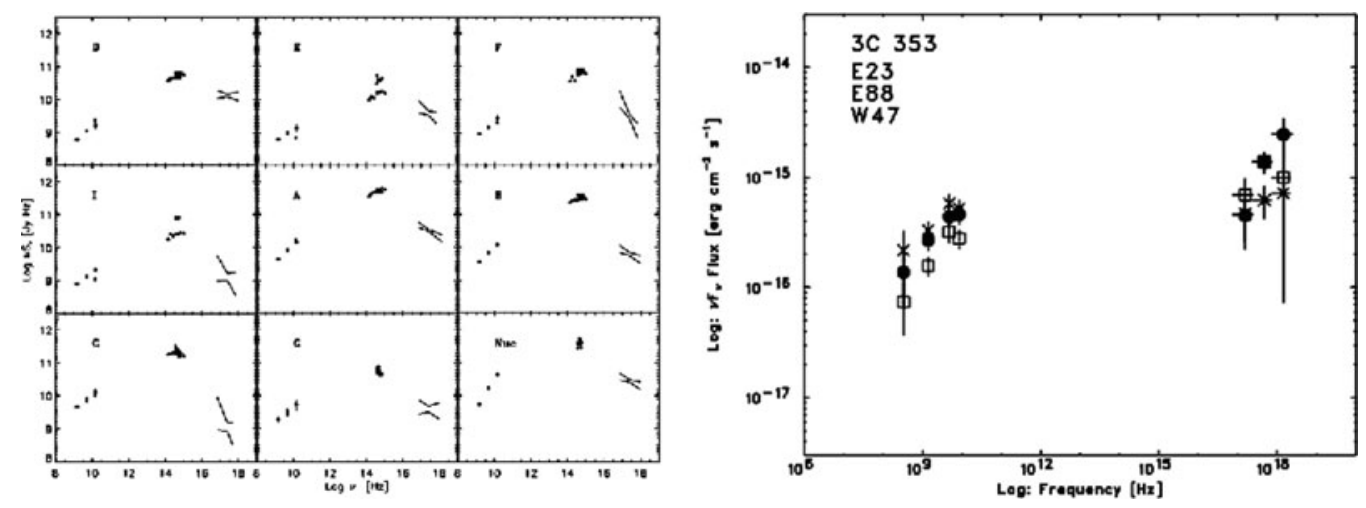

Figure 2. Example spectral energy distribution of jet knots in nearby FR I radio galaxy M 87 (left: figure from Marshall et al. 2002) and FR II radio galaxy 3C 353 (right: figure from Kataoka et al. 2008).

blazars, but also radio galaxies and possibly other classes of active galactic nuclei (AGNs) - with much improved sensitivity than previously available. For example, the second catalog of AGNs detected by the Fermi-LAT in two years of operation reported 886 gamma-ray sources located at high Galactic latitude $(|b|>10 \mathrm{deg})$ that are detected at $\simeq 5 \sigma$ significance level (Ackermann et al. 2011). These are mostly blazars but includes 8 misaligned AGNs, 4 NLSY1s and 10 AGNs of other types. This "misaligned AGN sample" includes seven Faranoff-Riley type I (low-power, hereafter FR I) radio galaxies, and only one Faranoff-Riley type II (high-power, hereafter FR II) radio sources. None of the X-ray bright Seyfert 1 galaxies to be detected in gamma-rays thus far (Kataoka et al. 2011, Ackermann et al. 2012). Four GeV-emitting radio galaxies are also recently discovered in TeV energies (M87; Acciari et al. 2008, Cen A; Aharonian et al. 2009, NGC 1275; Aleksic et al. 2012, and IC 310; Aleksic et al. 2010).

In this review, I will first summarize recent observational highlights of large scale jets in radio galaxies, specifically resolved by the Chandra X-ray observatory (section 2). Next I will move on to the gamma-ray sky to present some results from Fermi-LAT observations of "misaligned" blazars, namely radio galaxies (section 3). I will discuss a unification scheme connecting blazars and misaligned radio galaxies. Finally, I will make a short comment on recent multiband observations of the Fermi-bubble and possible analogy to the AGN jet physics (section 4).

\section{Misaligned AGN seen at X-rays}

The excellent spatial resolution of the Chandra X-Ray Observatory has opened a new era to study the large-scale jets in powerful extragalactic radio sources. Since the first memorial discovery of an X-ray jet in PKS 0637-752 (Chartas et al. 2000), more than 100 radio-loud AGNs are now known to possess X-ray counterparts to their radio jets, hot spots, or lobes on kpc to Mpc scales as of 2014 (see, Fig.1 for examples). Bright X-ray jet knots are most often detected, but the X-ray emissions from the hot spots and radio lobes are also reported in a number of FR II radio galaxies and quasars.

The broadband spectra of these jet-related structures, however, show great variety between radio and X-ray energy bands. In nearby FR I sources, the typical X-ray to-radio spectrum of the jet knots is consistent with a single, smoothly broken power-law continuum, suggesting that this broadband emission is entirely due to non-thermal synchrotron radiation from a single electron population (e.g., Marshall et al. 2002; Fig. 2 left). In 

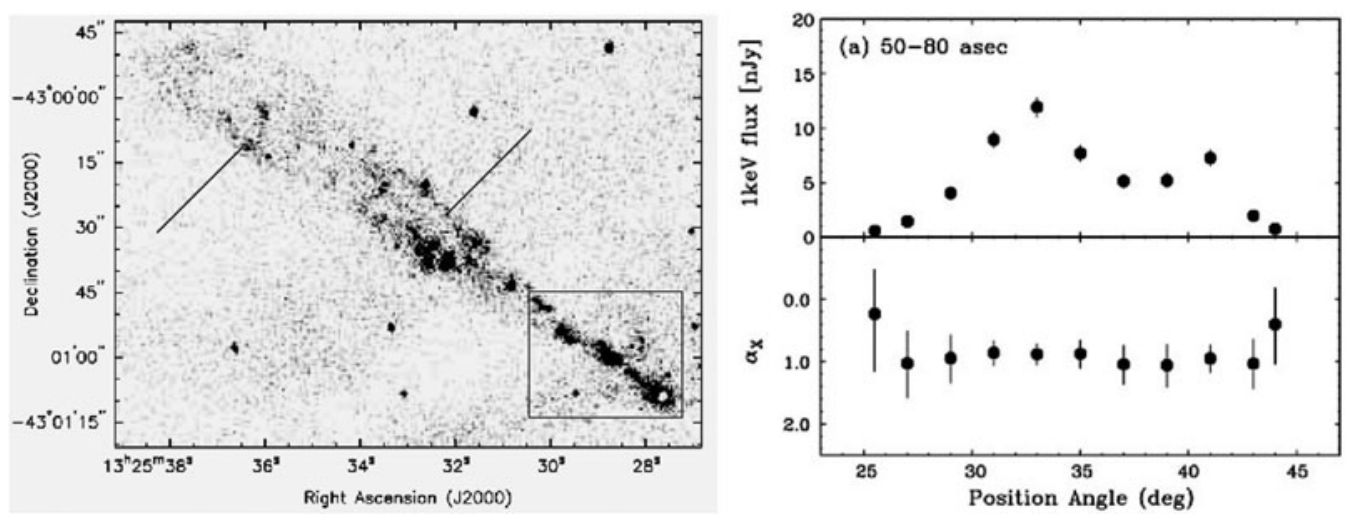

Figure 3. left; X-ray structure of the large scale jets in Cen A. The lines indicate two regions where the X-ray jet appears significantly edge brightened. Figure from Hardcastle et al. (2003). right; Transverse X-ray intensity profile measured at $1 \mathrm{keV}(t o p)$ and the energy spectral index (bottom). Figure from Kataoka et al. (2006).

most quasars, however, the X-ray knots' spectra are much harder than expected from a simple extrapolation of the radio-to-optical fluxes. In such situations, it is suggested that $\mathrm{X}$-ray photons are produced via the inverse Compton (IC) scattering of either synchrotron photons (SSC) or cosmic microwave background (CMB) photons (EC; Tavecchio et al. 2000, Celotti et al. 2001). On the other hand, such IC models cannot readily explain the detected X-ray emission from FR II jets characterized with large inclination angle (e.g., Fig.2 right; Wilson et al.2001; Kataoka et al. 2003, 2008) since they are expected to be the debeamed analogs of the radio-loud quasars. Alternative model involves the synchrotron emission from high-energy electrons characterized by "nonstandard" (concave) energy distribution (Stawarz \& Ostrowski 2002, Stawarz et al. 2004) or a separate distribution to the ones emitting the radio-to-optical continuum (Uchiyama et al. 2006).

In the case of the hot spots in powerful sources, one finds an analogous controversy regarding the X-ray emission: although in many objects this emission is consistent with the standard SSC model (see, e.g., Wilson et al. 2000), in some other sources it cannot be simply explained in this way, most likely suggesting a synchrotron origin of the detected X-ray photons (see, e.g., Hardcastle et al. 2004). For the extended lobes of quasars and FR II galaxies, it is established that the X-ray radiation is produced by the EC process involving CMB target radiation. In some cases, however, infrared target photons from quasar cores may contribute to the inverse Compton lobes' emission at keV photon energy ranges (e.g., Brunetti et al. 1997, Croston et al. 2005, Kataoka \& Stawarz 2005). In addition to these distinct structures (i.e., jet-knots, hot spots and lobes), extended emission all over the jet volume, most likely diffuse in nature, is found in nearby FR I radio galaxy Cen A (Kataoka et al. 2006, Worral et al. 2008). Interestingly, extended emission has a relatively flat-topped intensity profile in the transverse jet direction. In this context, many authors invoked external entrainment via a turbulent boundary layer, arising from Kelvin-Helmholtz (KH) instabilities at the jet boundary (De Young 1986, Bicknell et al. 1984, Bicknell et al. 1994). In fact, similar radial structures have been successfully described as turbulent, entraining, decelerating flows (e.g., 3C 31, NGC 315, or 3C 296; Laing et al. 2004, 2006a, 2006b) with the entrainment process strongly affecting the evolution of the source.

Finally, FR I radio galaxies are commonly found in the centers of rich galaxy groups and clusters and hence important to probe the interaction between the non-thermal jet 

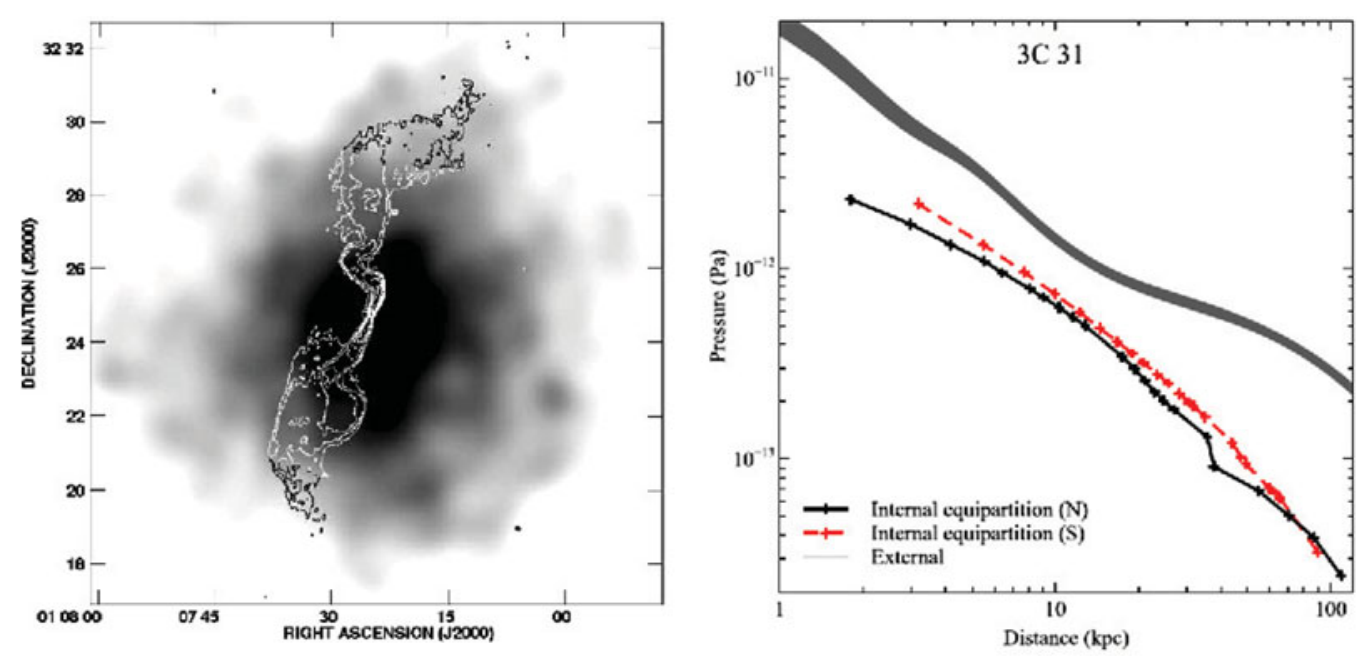

Figure 4. left; X-ray image of FRI radio galaxy 3C 31 taken by $X M M$-Newton in the $0.5-5$ $\mathrm{keV}$ energy range. Figure from Croston et al. (2008). right; external and internal pressure profiles for 3C 31. Figure from Croston \& Hardcastle (2014).

and external gas. The external pressure acting on the jets and lobes can be constrained tightly on scales of a few to several hundred kpc for many FR I radio galaxies, using X-ray observations of surrounding group or cluster gas with Chandra and XMM-Newton. In this way, Croston \& Hardcastle (2014) argue that the synchrotron-radiating particles and magnetic fields in FR I radio galaxies, if at equipartition, can provide only a small fraction of the total internal energy density of the radio lobes of 3C 31 and Hydra A (Fig.4). The entrainment of material from the jet surroundings can provide necessary pressure, and that the entrained material is heated to temperatures substantially about that of surrounding intracluster medium. Hence it seems that mass entrainment is important not only decelerating FR I jet but evolution and particle content of giant radio lobes. It is also suggested that giant lobes in FR I sources like Cen A are expected to be very turbulent and important site of ultra-high energy cosmic-ray (UHECR) acceleration via stochastic processes (Wykes et al. 2013).

\section{Misaligned AGN seen at Gamma-rays}

During the initial all-sky survey performed during the first 4 months after its launch, Fermi-LAT detected two brightest radio galaxies at high significance level $(>10 \sigma)$, namely, NGC 1275 (Abdo et al. 2009a) and Cen A (Abdo et al. 2009b, 2009c). The detection of $\mathrm{MeV} / \mathrm{GeV}$ emission from another famous radio galaxy $\mathrm{M} 87$ was reported based on 10 months of all-sky-survey Fermi-LAT data (Abdo et al. 2009d). Other radio galaxies are relatively faint in this respect, and detected later with improved photon statistics (Abdo et al. 2010a, Ackermann et al. 2011). Note that most of these "gammaloud" radio galaxies are nearby FR Is. The detection of NGC 1275 was particularly noteworthy because this source, unlike Cen A or M 87, was previously undetected in gamma-rays, neither by $C G R O / E G R E T$ during its $\sim 10$ year of operation, nor by groundbased Cherenkov telescopes. We however note that COS-B data taken between 1975 and 1979 (Strong et al. 198) showed a gamma-ray excess coincident with the position of this galaxy, and that broad correspondence between the high-frequency radio data the high-energy gamma-ray emission is apparent over four-decades light curve (Fig.5 

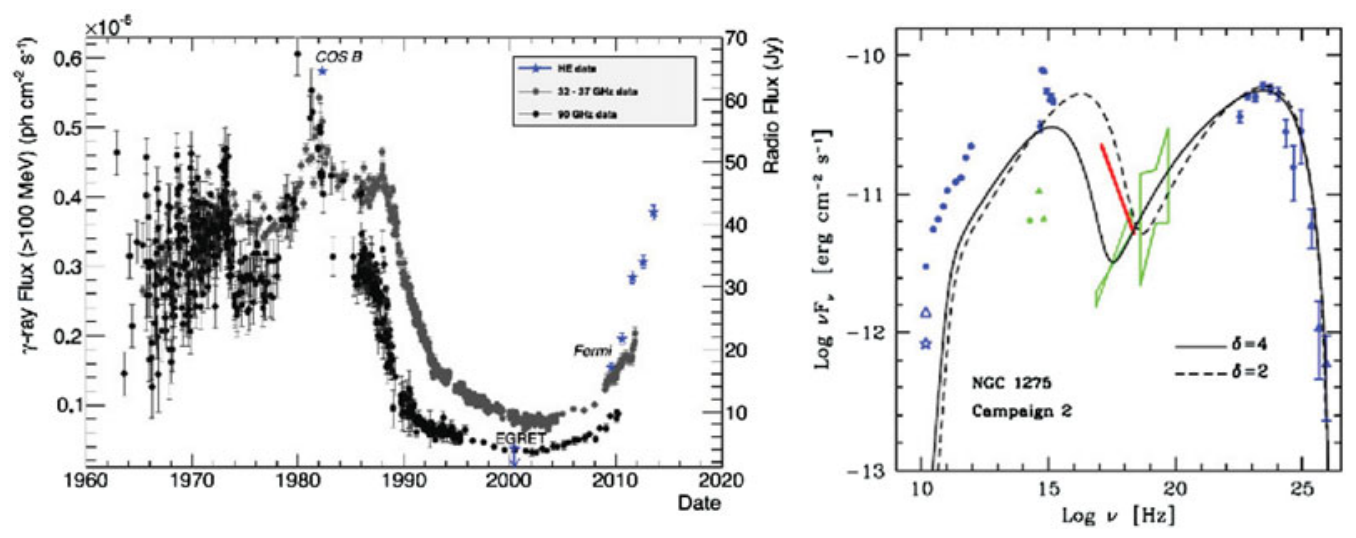

Figure 5. left; An historical light curve for NGC 1275, comparing a flux about $100 \mathrm{MeV}$ and radio flux between 32-90 GHz. Figure from Dutson et al. (2014). right; Spectral energy distribution of NGC 1275 between Aug. 2010 and Feb. 2011. Dashed and solid lines show the best-fit SSC models for a mild beaming factor $\delta=2$ or 4 . Figure from Aleksic et al. (2014).

left; Abdo et al. 2009a, Dutson et al. 2014). Besides prior TeV detection of Cen A and M 87, NGC 1275 was recently identified as a very high energy gamma-ray emitter (VHE; $>100 \mathrm{GeV}$ ) by MAGIC (Aleksic et al. 2012), leading to contemporaneous multi-frequency observations from radio to TeV energy (Aleksic et al. 2014).

Fig. 5 (right) shows the overall SED of NGC 1275 constructed with radio to gammaray multiband data (Aleksic et al. 2014; but see also, Abdo et al. 2009a), which appears to be very similar to classical TeV-emitting AGNs (like Mrk 421 and Mrk 501) but peaking at relatively low energy. Following an initial attempts of SED modeling by Abdo et al. (2009a), a simple one-zone SSC model yields a jetted outflow with bulk Lorentz factor $\Gamma=1.8$ and Doppler factor $\delta=2.3$, so that the observing angle to the jet direction is $\theta=25^{\circ}$. The total jet power would be $10^{44-45} \mathrm{erg} / \mathrm{s}$. The mean magnetic field in the radiating plasma is $B=0.05 \mathrm{G}$, and the comoving radius of the jet emission region is $2 \times 10^{18} \mathrm{~cm}$, corresponding to a variability timescale of $\approx 1 \mathrm{yr}$. This simple homogeneous model provides an adequate fit the NGC 1275 data, and is consistent with mildly relativistic outflows observed in the expanding radio lobe of $3 \mathrm{C} 84$ situated at the center of NGC 1275 (Asada et al. 2006). In the context of BL Lac and FR I unification, models with structure jets involving decelerating flows (see, Abdo et al. 2009a) or a spine-sheath model (Ghisellini et al. 2005; see also Section 2), make predictions for FR I radio galaxies as potential Fermi gamma-ray sources. Indeed, Ghisellini et al. (2005) predicted that 3C 84 (i.e., NGC 1275) would be one of the strongest gamma-ray emitting radio galaxies above $100 \mathrm{MeV}$.

Next topic concerning the Fermi-LAT detection of radio galaxies is the discovery of gamma-ray emission from two broad line radio galaxies (BLRGs). BLRGs seem ideal targets for an in-depth investigation of radio-loud/quiet AGNs, since this particular class of very radio-loud AGN exhibits both the disk-related ('Seyfert-like') and the jet-related ('blazar-like') radiative signatures in their broad-band spectra. Unlike blazars, the jets in BLRGs are not pointing directly toward the observer, and so the relativistic beaming effects and the related jet dominance are only moderate. Moreover, unlike narrow-line radio galaxies (NRLGs), BLRGs are not generally obscured by large amounts of dust distributed in torus-like structures around the nucleus, and hence radiative properties of the accretion disks and of the circumnuclear gas can be easily accessed in their case. 

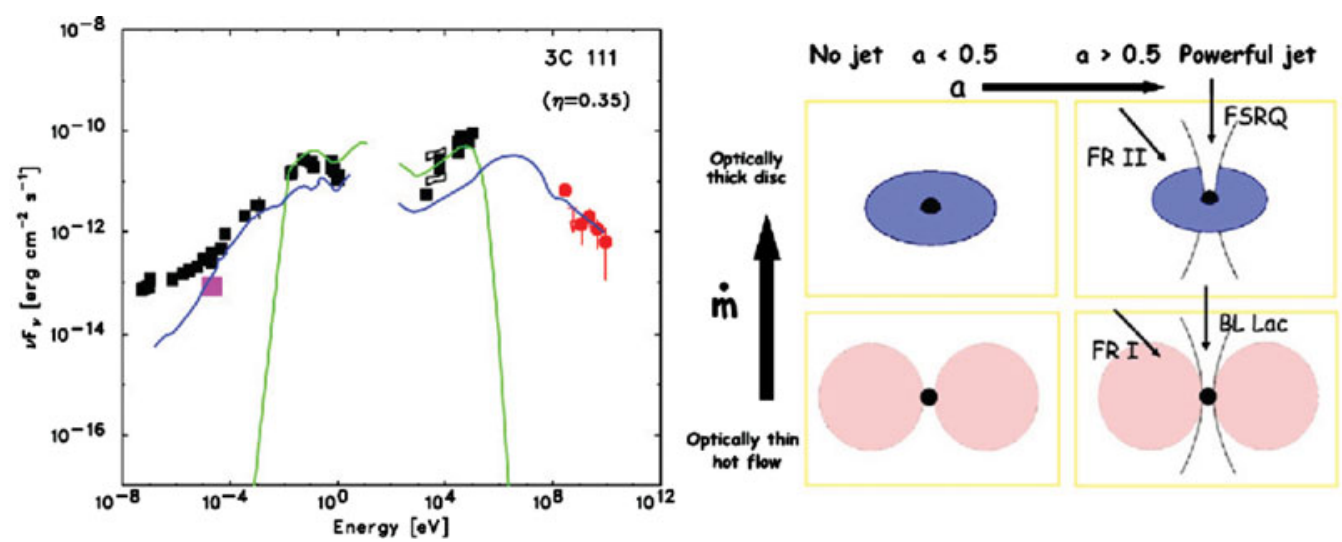

Figure 6. left; Broadband SED of BLRG 3C 111 detected by Fermi-LAT. The green curve corresponds to the template of accretion-related Seyfert-type emission and the blue curve corresponds to the non-thermal blazar-type jet emission. Figure from Kataoka et al. (2011). right; Grand unification scheme for AGN in terms of different black hole spin and accretion rate. Figure from Maraschi et al. (2012).
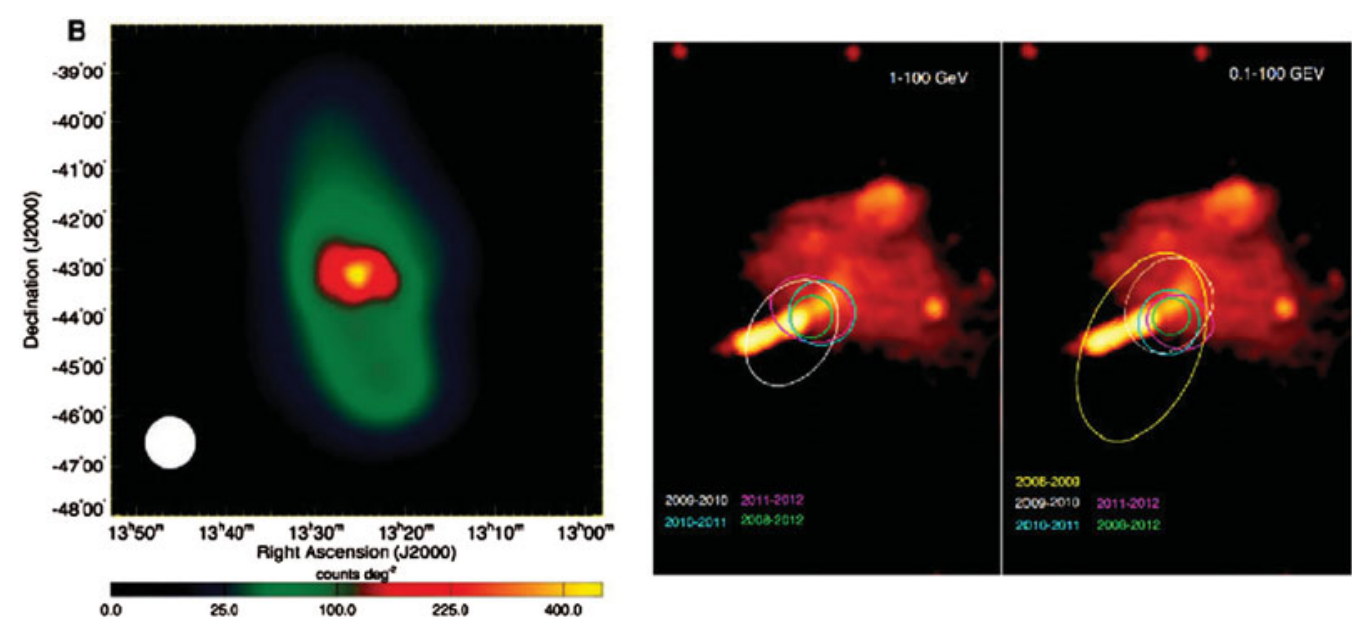

Figure 7. left; Fermi-LAT gamma-ray $(>200 \mathrm{MeV})$ countmaps of Cen A after subtraction of field point sources. Figure from Abdo et al. (2010b). right; NGC 6251 radio map at $92 \mathrm{~cm}$. Ellipses represents the best-fit position (68\% uncertainty) of the gamma-ray source. Figure from Grandi et al. (2013).

Several authors in the past speculated about the non-negligible jet contribution to the X-ray emission of BLRGs, diluting the accretion-related radiative output in the X-ray domain (Wozniak et al. 1998, Eracleous et al. 2000, Grandi et al. 2002). This idea was subsequently examined in various different approaches using most recent broadband Xray data obtained with BeppoSAX (Grandi et al. 2006; Grandi \& Palumbo 2007), Suzaku and Swift (Kataoka et al. 2007, Sambruna et al. 2009).

Fig. 6 (left) presents SED of LAT-detected BLRG 3C 111. To reproduce the overall spectral feature, a simple phenomenological "hybrid" model, consisting of individual thermal (i.e., Seyfert-like) and non-thermal (i.e., blazar-like) emission components are considered (see, Kataoka et al. 2011 for more details). Note that gamma-ray detected BLRGs, 3C 111 and 3C 120, show the strongest nuclear flux in the radio band. This 

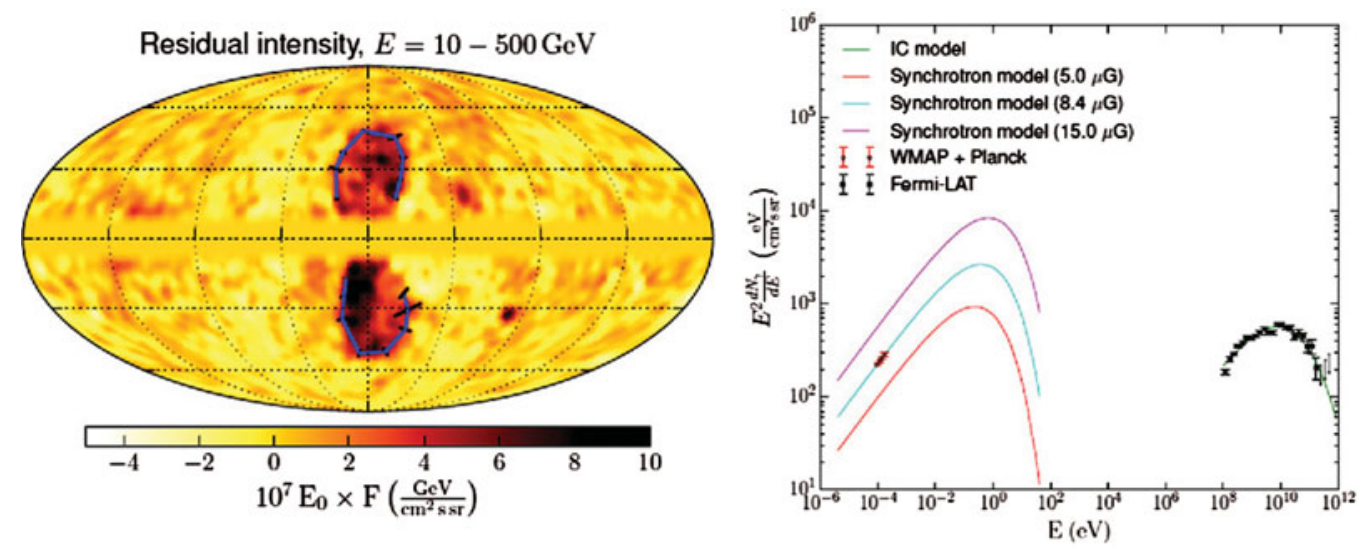

Figure 8. left; Residual gamma-ray map overplotted with the edge of the bubbles. right; SED of Fermi Bubbles modeled with IC and synchrotron emission from the same population of electrons. Both figures from Ackermann et al. (2014).

suggests that the GeV emission of BLRGs is most likely dominated by the beamedradiation of relativistic jets observed at intermediate viewing angles. Also, analysis of a comparison sample of high-accretion-rate Seyfert 1 galaxies, which can be considered radio-quiet counterparts of BLRGs, suggests that the relative contribution of the nuclear jets to the accreting matter is $>1 \%$ on average for BLRGs, whereas it is $<0.1 \%$ for Seyfert 1 galaxies. In this context, a role of black hole spin and accretion in powering relativistic jets in AGNs are extensively discussed in Maraschi et al. (2012), leading to a grand unification of AGN based on two intrinsic parameters of spin and accretion rate in Eddington units (Fig. 6 right). More recently, Sikora et al. (2013) found a strong negative correlation between $\lambda$ and $R$, where $\lambda$ is the Eddington ratio and $R$ is the radioloudness parameter. This may favor a jet production model which involves the so-called magnetically choked accretion scenario (Narayan et al. 2003).

Finally, we should remark that Fermi-LAT provides precise gamma-ray localizations of typically 2' -5 ', which corresponds to $\sim 10 \mathrm{kpc}$ for nearby sources at the distance of 100 Mpc. This means that gamma-ray emission site in the jet or spatial extent of radio lobe, if exist, may be directly visible for the first time. In fact, LAT has detected the gammaray glow emanating from the giant lobes of Cen A (Abdo et al. 2010b, Yang et al. 2012). The resolved gamma-ray images shows the lobes clearly separated from the central AGN as discussed above (Fig. 7 left). As indicated from the X-ray observations of radio-lobe, the gamma-ray emission from the lobes is naturally interpreted as IC emission of CMB photons, with possible other contributions from extragalactic background light. Again, investigation of possible relation to UHECR is very interesting topic in the near future (Wykes et al. 2013). Also it has been suggested that gamma-ray emission of NGC 6251 peaks on the jet at $7 \pm 3$ ' (or $204 \pm 87 \mathrm{kpc}$ ) from the radio core, whilst the source clearly exhibits temporal variability of a sub-year scale (Grandi et al. 2013). This reminds us significant flare of HST-1 in the M87 jet observed with Chandra (Harris et al. 2003) that may be connected with the $\mathrm{TeV}$ flare detected in 2005. Similarly, multiple zone in the jet could then contribute to the high energy emission of NGC 6251 but further study is necessary to confirm this. 


\section{Fermi Bubbles - nearest AGN in the Galactic Center?}

Finally, we note an interesting analogy between the the giant lobe as discussed above in Cen A, and "Fermi Bubbles", extending for about $50 \mathrm{deg}$ (or $8.5 \mathrm{kpc}$ ) about and below the Galactic Center (GC) (Dobler et al. 2010, Su et al. 2010, Ackermann et al. 2014; Fig. 8 left). The gamma-ray emission of the bubbles is spatially correlated with the flatspectrum microwave excess known as the "WMAP haze", as is the case in Cen A lobe. Hence it has been argued that the WMAP haze and the Fermi bubbles are linked, and that the gamma-ray emission of the bubbles is simply the inverse-Compton scattering of the $\mathrm{CMB}$ (IC/CMB) emission from the same electron population producing the observed microwave synchrotron excess. At X-ray frequencies, hints of signatures of the entire north bubble structure can be noted in the ROSAT map, particularly a prominent Galactic feature called the North Polar Spur (NPS) seen in the $0.75 \mathrm{keV}$ (R45 band) map (Su et al. 2010, Kataoka et al. 2013). Sofue (2000, and references therein) interpreted the NPS as a large scale outflow from the GC with a corresponding total energy of $\sim 10^{55-56} \mathrm{erg}$ released on a timescale of $\sim 10 \mathrm{Myr}$, almost comparable with what observed in nearby radio galaxies. Fig. 8 (right) shows the SED of the Fermi Bubbles, from radio to GeV gamma-ray, as detailed in literature (Ackermann et al. 2014; see also Kataoka et al. 2013). The non-thermal pressure and energy estimated from a one-zone leptonic model are in rough equilibrium with that of the surrounding thermal plasma. In this context, the recent Suzaku observations of Cen A lobe indicated analogously a rough pressure equilibrium with the surrounding medium (Stawarz et al. 2013). In both cases, the radiating ultrarelativistic electrons were proposed to be accelerated $i n$-situ via interaction with magnetic turbulence, rather than at weak shocks formed eventually at the bubbles/lobes edges.

Not only Fermi Bubbles, but there are number of observations discussed in the literature taken as evidence that the GC has undergone multiple past epochs of enhanced activity on different timescales, due either to AGN-like outbursts or episodes of circumnuclear starbursts (for a review, see, $\mathrm{Su}$ et al. 2010, Kataoka et al. 2013). Outflows of this kind are expected to lead to the formation of bubbles/lobes expanding within the Galactic halo, just like the GC scenario of NPS as well as the Fermi Bubbles, sweeping up the interstellar/halo gas in analogy with the extended lobes seen in distant radio galaxies Sofue 2000. High-quality radio and X-ray observations of the Fermi Bubbles interiors, enabling a diagnosis of the plasma conditions and magnetic field structure similarly as in the case of the giant Cen A lobes are needed to elaborate more on the particle acceleration process at work.

\section{Conclusion}

In this paper, I quickly reviewed observational highlights and recent progress in AGN jet studies at X-ray and gamma-ray energy bands.

- Chandra X-ray observatory reveals more than 100 large scale X-ray jets in misaligned radio galaxies. "Structured jet" consisting of fast spine and slow sheath is also supported from X-ray observations.

- Not only blazars but misaligned AGNs are also being important gamma-ray emitter as detected by Fermi-LAT. Some new hints/ideas toward grand unification between radio-loud and radio-quiet AGNs now being discussed.

- Further new surprise is the detection of gamma-ray lobes in Cen A, together with Fermi Bubbles in our Galaxy. Possible links between AGN and normal galaxy will be studied further. 


\section{References}

Abdo, A., et al. 2009a, ApJ, 699, 31

Abdo, A., et al. 2009b, ApJ, 700, 597

Abdo, A., et al. 2009c, ApJS, 183, 46

Abdo, A., et al. 2009d, ApJ, 707, 55

Abdo, A., et al. 2010a, ApJ, 715, 429

Abdo, A., et al. 2010b, Science, 328, 725

Abramowicz, M. A., Chen, X., Kato, S., Lasota, J.-P., \& Regev, O. 1995, ApJ, 438, L37

Acciari, V. A., et al. 2008, ApJ, 679, 397

Ackermann, M. et al. 2011, ApJ, 743, 171

Ackermann, M, et al. 2012, ApJ, 747, 104

Ackermann, M. et al. 2014, ApJ, 793, 64

Aharonian, F., et al. 2009, ApJ, 695, L40

Aleksic, J., et al. 2010, ApJ, 723, L207

Aleksic, J., et al. 2012, A\&A, 539, L2

Aleksic, J., et al. 2014, $A \mathscr{E} A, 564,5$

Antonucci, R. 1993, ARAEA A, 31, 473

Asada, K., et al. 2006, PASJ, 58, 261

Barthel, P. D. 1989, ApJ, 336, 606

Begelman, M. C., Blandford, R. D., \& Rees, M. J. 1984, Rev. Mod. Phys., 56, 255

Bicknell, G. V. 1984, ApJ, 286, 68

Bicknell, G. V. 1994, ApJ, 422, 542

Blandford, R. D. \& Znajek, R. L. 1977, MNRAS, 179, 433

Blandford, R. D. 1990, in Active Galactic Nuclei, ed. T. J.-L. Courvoisier \& M. Mayor (Berlin: Springer), 161

Bridle, A. H. \& Perley, R. A. 1984, ARA\&A, 22, 319

Brunetti, M., et al. 2004, ApJ, 612, 729

Celotti, A., et al. 2001, MNRAS, 321, L1

Chartas, G, et al. 2000, ApJ, 542, 655

Croston, J. H., et al. 2005, ApJ, 626, 733

Croston, J. H., et al. 2008, MNRAS, 386, 1709

Croston, J. H. \& Hardcastle, M, J. 2014, MNRAS, 438, 3310

De Young, D. S. 1986, ApJ, 307, 62

Dobler, G., et al. 2010, ApJ, 717, 825

Dutson, K. L., et al. 2014, MNRAS, 442, 2048

Eracleous, M., Sambruna, R., \& Mushotzky, R. F. 2000, ApJ, 537, 654

Fabian, A. C. \& Rees, M. J. 1995, MNRAS, 277, L55

Garofalo, D. 2009, ApJ, 699, 400

Ghisellini, G., Tavecchio, F., \& Chiaberge, M., 2005, A\&SA, 432, 401

Ghisellini, G., Maraschi, L., \& Tavecchio, F. 2009, MNRAS, 396, L105

Grandi, P., Urry, C. M., \& Maraschi, L. 2002, New Astron. Revs, 46, 221

Grandi, P., Malaguti,G. \& Fiocchi, M., 2006, ApJ, 642, 113

Grandi, P., \& Palumbo, G. G., 2007, ApJ, 659, 235

Grandi, P., et al. 2013, EPJ Wev of Conference, 61, 04007

Hardcastle, M. J. et al. 2003, ApJ, 593, 169

Hardcastle, M. J., Harris, D. E., Worrall, D. M., \& Birkinshaw, M. 2004, ApJ, 612, 729

Harris, D. E. \& Krawczynski, H. 2002, ApJ, 565, 244

Harris, D. E., et al. 2003, ApJ, 586, L41

Harris, D. E. \& Krawczynski, H. 2006, ARA\&A, 44, 463

Kataoka, J., et al. 2003, A\&A, 399, 91

Kataoka, J. \& Stawarz, Ł. 2005, ApJ, 622, 797

Kataoka, J., et al. 2006, ApJ, 641, 158

Kataoka, J., et al. 2007, PASJ, 59, 279

Kataoka, J., et al. 2008, ApJ, 685, 839 
Kataoka, J., et al. 2011, ApJ, 740, 29

Kataoka, J., et al. 2013, ApJ, 779, 57

Koide, S., Shibata, K., Kudoh, T., \& Meier, D. L. 2002, Science, 295, 1688

Komossa, S. 2008, RevMexAA Conf. Ser., 32, 86

Kukula, M. J., Pedlar, A., Baum, S. A., \& O'Dea, C. P. 1995, MNRAS, 276, 1262

Laing, R. A. \& Bridle, A. H.,. 2004, MNRAS, 348, 1459

Laing, R. A., et al. 2006a, MNRAS, 372, 510

Laing, R. A., et al. 2006b, MNRAS, 368, 48

Lasota, J.-P. 1996, in Int. Workshop on Basic Physics of Accretion Disks, Advection-dominated Galactic Nuclei, ed. S. Kato et al. (Amsterdam: Gordon and Breach), 85

Maraschi, L., Colpi, M., Ghisellini, G., Perego, A., \& Tavecchio, F. 2012, Journal of Physics Conference Series, 355, 012016

Marshall, H, L., et al. 2002, ApJ, 564, 683

Massaro, F., Harris, D. E., \& Cheung, C. C. 2011, ApJS, 197,24

Massaro, F., et al. 2012, ApJS, 203, 31

Middelberg, E., Roy, A. L., Nagar, N. M., et al. 2004, A\&̈A, 417, 925

Narayan, R. \& Yi, I. 1994, ApJ, 428, L13

Narayan, R. \& Yi, I. 1995, ApJ, 444, 231

Narayan, R., Igumenshchev, I, V., \& Abramowicz, M, A., 2003, PASJ, 55, L69

Pogge, R. W. 2000, New Astron. Revs, 44, 381

Sambruna, R. M., et al., 2009, ApJ, 700, 1473

Shakura, N. I. \& Sunyaev, R. A. 1973, A\& A, 24, 337

Sikora, M., Stawarz, L., \& Lasota, J.-P. 2007, ApJ, 658, 815

Sikora, M., et al., 2013, ApJ, 765, 62

Sofue, Y. et al., 2000, ApJ, 540, 224

Stawarz, L, Ostrowski, M. 2002, ApJ, 578, 763

Stawarz, L., et al. 2004, ApJ, 608, 95

Stawarz, L., et al. 2013, ApJ, 766, 48

Strong, A. W., et al. 1982, A\&A, 115, 404

$\mathrm{Su}$, M., Slatyer, T. R., \& Finkbeiner, D. P., 2010, ApJ, 724, 1044

Tavecchio, F., et al. 2000, ApJ, 544, L23

Tchekhovskoy, A., Narayan, R., \& McKinney, J. C. 2010, ApJ, 711, 50

Thean, A. H. C., et al.. 2001, MNRAS, 327, 369

Uchiyama, Y., et al. 2006, ApJ, 648, 910

Ulvestad, J. S. \& Wilson, A. S. 1989, ApJ, 343, 659

Urry, C. M. \& Padovani, P. 1995, PASP, 107, 803

White, R. L., Becker, R. H., Gregg, M. D., et al.. 2000, ApJS, 126, 133

Wilson, A. S., et al. 2000, ApJ, 544, L27

Wilson, A. S., Young, A, J., \& Shopbell, P. L. 2001, ApJ, 547, 740

Worrall, D. M., et al. 2008, ApJ, 673, L135

Wozniak, P. R., et al. 1998, MNRAS, 299, 449

Wykes, S., et al. 2013, A\& $A, 558,19$

Yang, R. Z., et al. 2012, A\&A, 541, 19 